\title{
Influence of antiferroelectric-like behavior on tuning properties of ferroelectric HZO-based varactors
}

\author{
Sukhrob Abdulazhanov ${ }^{1}(1) \cdot$ Maximilian Lederer ${ }^{1} \cdot$ David Lehninger $^{1} \cdot$ Tarek Ali $^{1} \cdot$ Jennifer Emara ${ }^{1} \cdot$ Ricardo Olivo $^{1}$. \\ Thomas Kämpfe ${ }^{1}$
}

Received: 5 May 2021 / Accepted: 4 July 2021 / Published online: 23 July 2021

(c) The Author(s) 2021

\begin{abstract}
In this article, we investigate the capacitance-voltage (C-V) characteristics of $\mathrm{Hf}_{x} \mathrm{Zr}_{1-x} \mathrm{O}_{2}$ metal-ferroelectric-metal (MFM) thin-film capacitors with various $\mathrm{Zr}$ doping for varactor applications. The impact of field cycling during the wake-up process on the capacitance was analyzed. In addition, the effect of antiferroelectric-like (AFE) behavior on tuning was investigated. The transition between ferroelectric (FE) and AFE regime is particularly interesting for varactor application, as a reduced bias is required for tuning. The cycle dependence of the FE and AFE properties at elevated temperatures was also investigated, where it was shown that with an increase of temperature, the tunability is reduced. Temperature measurements also comply with recent studies of ferroelastic nature of AFE behavior.
\end{abstract}

\section{Introduction}

With the progress in wireless networks, including the upcoming $5 \mathrm{G}$ and Internet of Things, there is a high concern in exploring materials for passive RF devices. The main criteria are good CMOS compatibility, low required tuning voltage, high scalability, and low power consumption. Ferroelectric materials, like barium strontium titanate (BST) [1], have been widely used as tunable thin-film capacitors - varactors. However, they lack CMOS compatibility and require relatively high tuning voltages. Hafnium zirconium oxide (HZO) is a fully CMOS-compatible material [2], with a good back-end-of-line integration possibility due to its low thermal budget [3]. It is a good candidate for varactor applications [4], with a high miniaturization possibility $[5,6]$.

\section{Experimental}

The HZO MFM stacks were fabricated on top of highly doped $300 \mathrm{~mm}$ silicon wafers, which were meant to act as a conducting substrate for latter electrical measurements. The fabrication was carried out in several steps. First, the bottom titanium nitride (TiN) electrode of $10 \mathrm{~nm}$ thickness was deposited by means of atomic layer deposition (ALD). Afterwards, the HZO layer was deposited by ALD with $\mathrm{HfCl}_{4}$ and $\mathrm{ZrCl}_{4}$ precursors, and $\mathrm{H}_{2} \mathrm{O}$ as the oxidizing reactant. The $\mathrm{Hf}$ and $\mathrm{Zr}$ precursor cycling ratios were chosen to be $1: 1$ and 3:5, and the film thickness was set to $10 \mathrm{~nm}$ (Fig. 1a). On top of HZO, a $10 \mathrm{~nm}$ capping TiN electrode was deposited by physical vapor deposition (PVD), namely reactive magnetron sputtering. For the HZO crystallization, the samples were annealed by a rapid thermal anneal (RTA) at $450{ }^{\circ} \mathrm{C}$ for $60 \mathrm{~s}$ in $\mathrm{N}_{2}$ atmosphere. Finally, for the further electrical measurements, the Ni/Pt dot contacts were sputtered on top of the MFM stacks by using an electron beam evaporation through a shadow mask. The dots also played a role of a hard mask for a wet-etching of the top TiN layer with $\mathrm{SC} 1$ solution $\left(\mathrm{NH}_{4} \mathrm{OH}+\mathrm{H}_{2} \mathrm{O}_{2}\right)$ and thereby forming the MFM capacitors (Fig. 1b).

Structural characterizations of obtained MFM stacks were performed with a high-resolution Grazing Incidence X-ray diffraction (GIXRD) (Fig. 1c). The $2 \Theta$ angle was varied between 23 and $45^{\circ}$. The diffractograms reveal the crystallization of $\mathrm{HZO}$ with both 1:1 and 3:5 doping ratios into an orthorhombic/tetragonal phase.

Sukhrob Abdulazhanov

sukhrob.abdulazhanov@ipms.fraunhofer.de

1 Fraunhofer IPMS - Center Nanoelectronic Technologies (CNT), An der Bartlake 5, 01109 Dresden, Germany 


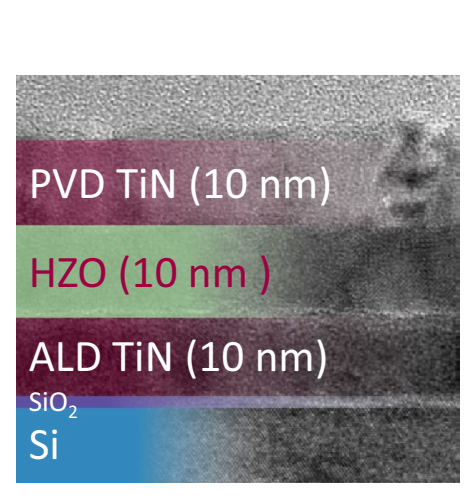

(a)

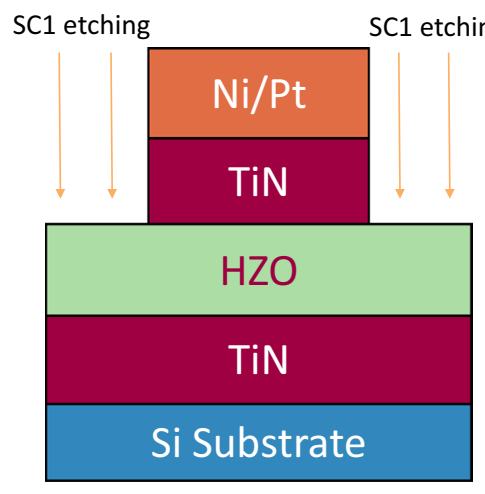

(b)

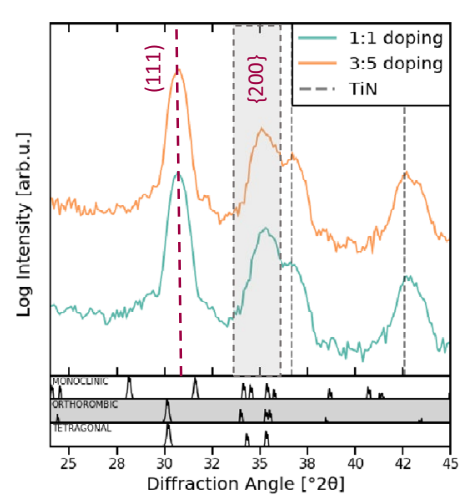

(c)

Fig. 1 TEM image (a) and the high-resolution GIXRD diffractogram (c) of the fabricated MFM stack with different hafnium-to-zirconium doping ratios; (b) cross-section schematics of a fabricated varactor

\section{Electrical characterization}

All electrical measurements were performed with an Aixacct TF 3000 analyzer. For standard I-V and P-V characteristics, a triangular waveform with $1 \mathrm{kHz}$ frequency and $3.3 \mathrm{~V}$ amplitude was used (Fig. 2). $\mathrm{C}-\mathrm{V}$ measurements were conducted with a small-amplitude $(50 \mathrm{mV})$ sinusoidal signal of $1 \mathrm{kHz}$ frequency, upon sweeping of DC bias between -3.3 and $3.3 \mathrm{~V}$ (Fig. 3). For an electrical field cycling during the wake-up treatment, a $20 \mathrm{~Hz}$ signal of a square waveform with an amplitude of $3.3 \mathrm{~V}$ was applied. Such a low frequency was chosen in order to better observe the development of electrical characteristics between the cycles. To obtain a full wake-up, the samples were cycled up to 3000-3600 times. In the case of temperature measurements (Fig. 4), the frequency of the I-V signal was reduced to $100 \mathrm{~Hz}$ for a smoother peak generation, and an amplitude of all measurements, including the cycling, was reduced to $2.6 \mathrm{~V}$ to avoid a breakdown at elevated temperatures. Diameters of the measured samples vary between 100 and $106 \mu \mathrm{m}$.
Fig. 2 I-V (a, b) and P-V (c, d) characteristics of $\mathrm{HZO}$, which undergoes the wake-up process, with 1:1 and 3:5 Hf:Zr doping ratios, respectively

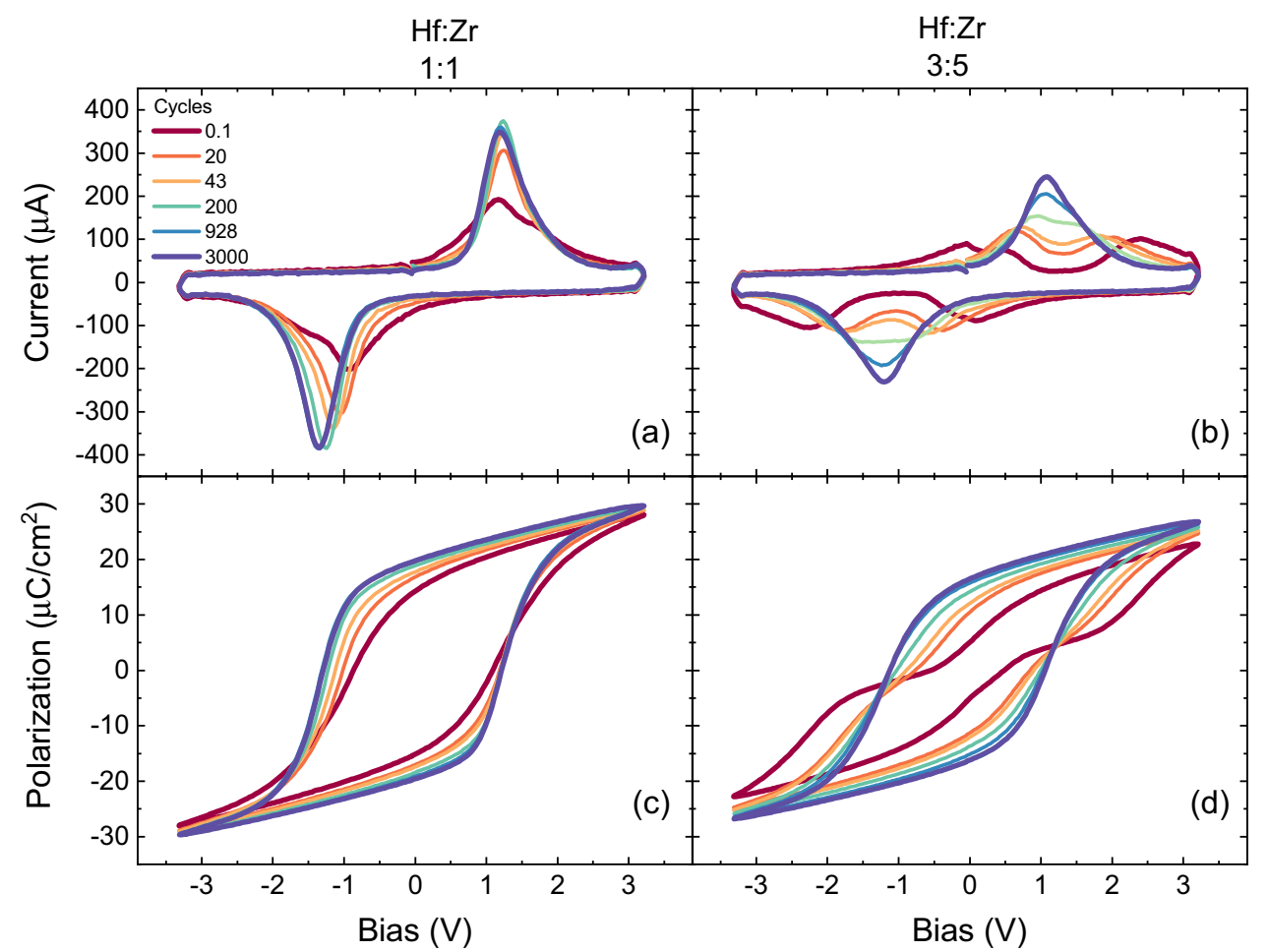




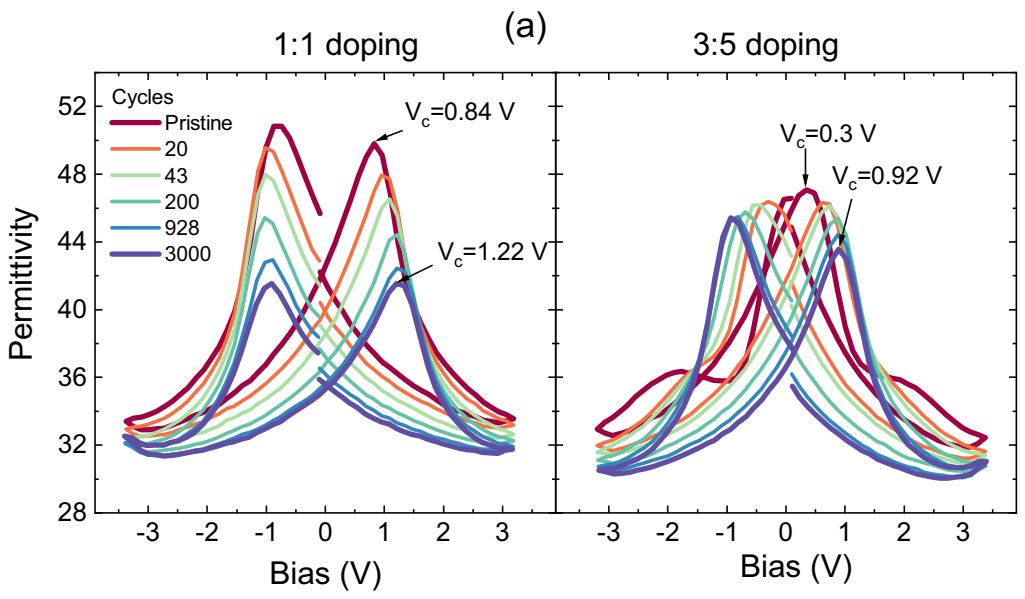

(b)

(c)
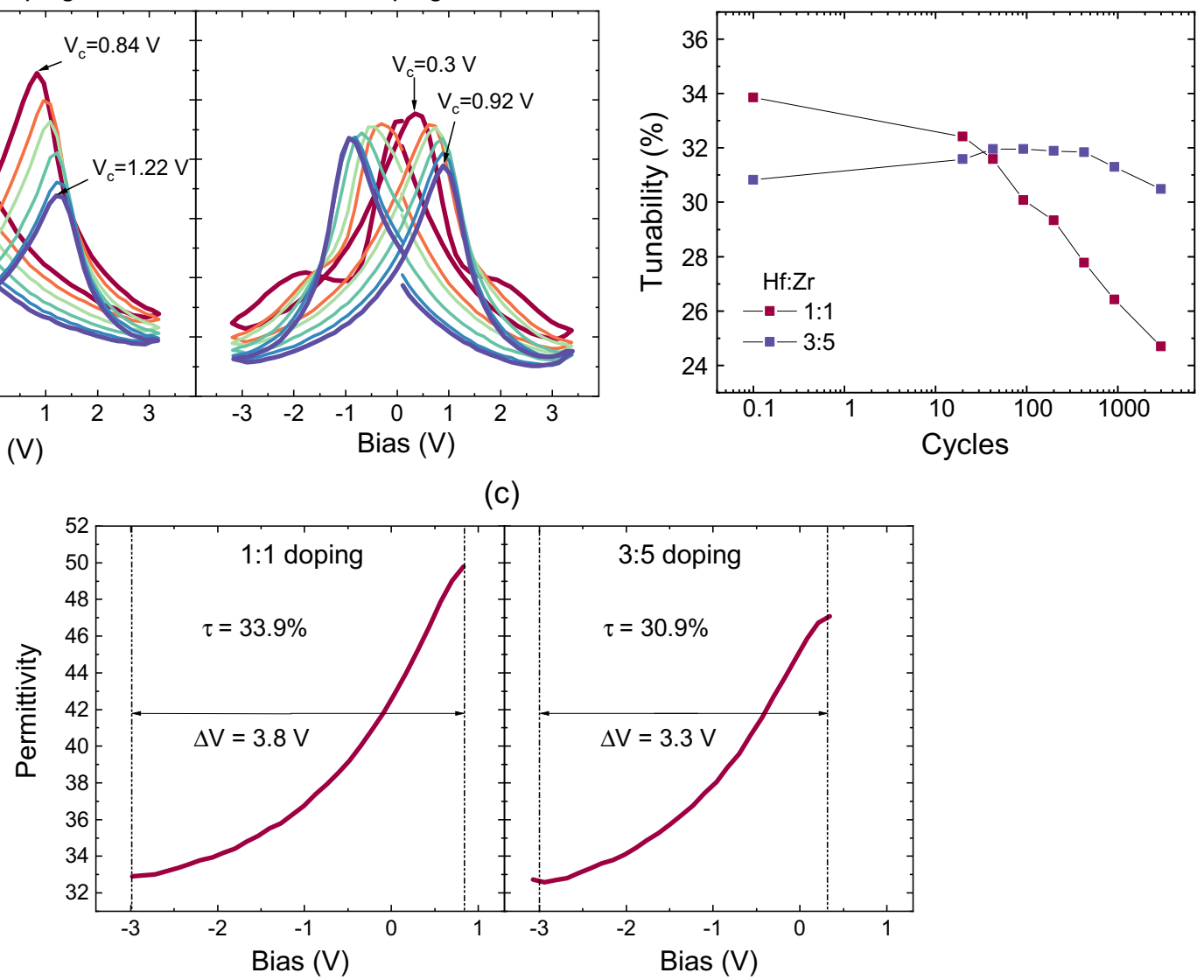

Fig. 3 Development of C-V characteristics (a) and tunability (b) of 1:1 and 3:5 HZO upon the wake-up cycling; (c) C-V characteristics and tuning range during the operation in the varactor mode

\section{Results and discussion}

In Fig. 2, a standard wake-up process is demonstrated. The wake-up is required for a full enhancement of the FE behavior. The peaks in the $\mathrm{I}-\mathrm{V}$ curves (Fig. 2a, b) represent the displacement currents upon the reorientation of a polarization axis of domains. In the case of $1: 1$ doped HZO films (Fig. 2a), which show dominating FE behavior, the I-V peak in a pristine state is slightly broadened. This is due to inhomogeneous distribution of coercive fields [7]. Upon wake-up, a pinched I-V peak increases in the amplitude and confines itself into a single peak with a higher amplitude at a certain coercive field $\left(\mathrm{V}_{\mathrm{c}}\right)$, which in turn manifests itself in the broadening of a $\mathrm{P}-\mathrm{V}$ hysteresis and increasing of remanent polarization $\left(\mathrm{P}_{\mathrm{r}}\right)($ Fig. $2 \mathrm{c})$. In the case of $\mathrm{HZO}$ films with 3:5 Hf:Zr ratio, the I-V characteristics show two separated peaks in the pristine state (Fig. 2b), representing the pinched AFE characteristic switching, resulting in a double hysteresis loop (Fig. 2d). Upon wake-up, the peaks are moving towards $\mathrm{V}_{\mathrm{c}}$, overlap with each other, and the AFE behavior develops into a strong FE behavior (Fig. 2d). The origin of such a behavior was briefly explained by [8-10], where the mechanism of AFE behavior was attributed to the presence of in-plane-oriented domains in combination with ferroelastic switching. Thus, the I-V peak located on a lower bias can be assigned to an in-plane domain orientation, and the one at a higher bias is responsible for the orientation in out of plane.

In Fig. 3a, the evolution of the $\mathrm{C}-\mathrm{V}$ characteristics upon wake-up of $\mathrm{HZO}$ with 1:1 and 3:5 doping ratio is shown. It can be seen that the $\mathrm{C}-\mathrm{V}$ peaks are shifting towards the coercive voltage in a similar way as for the I-V curves; thus, they are of the same origin and represent the domains with certain orientation. When operating in a varactor mode, the bias is varied between the maximum value $V_{\max }$ and $V_{c}$ (Fig. 3c). The varactor's tunability is defined as follows:

$\tau=\frac{\varepsilon_{r}\left(V_{c}\right)-\varepsilon_{r}\left(V_{\text {max }}\right)}{\varepsilon_{r}\left(V_{c}\right)}$,

where $\varepsilon_{r}\left(V_{c}\right)$ is permittivity at coercive voltage and $\varepsilon_{r}\left(V_{\text {max }}\right)$ is permittivity at maximum tuning voltage. 
(a)

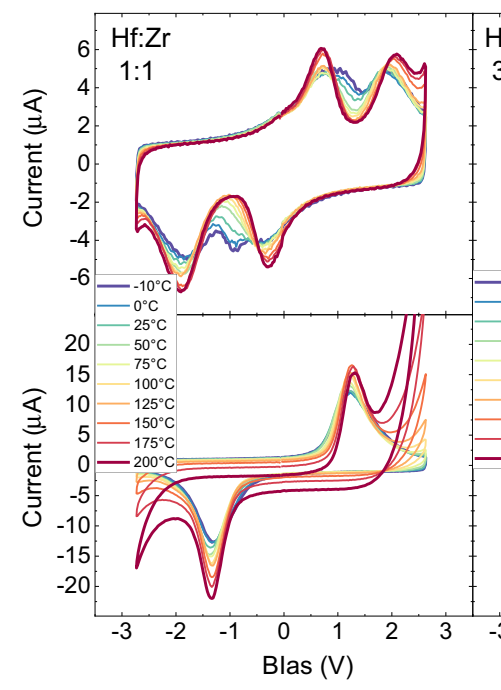

\section{Hizr}

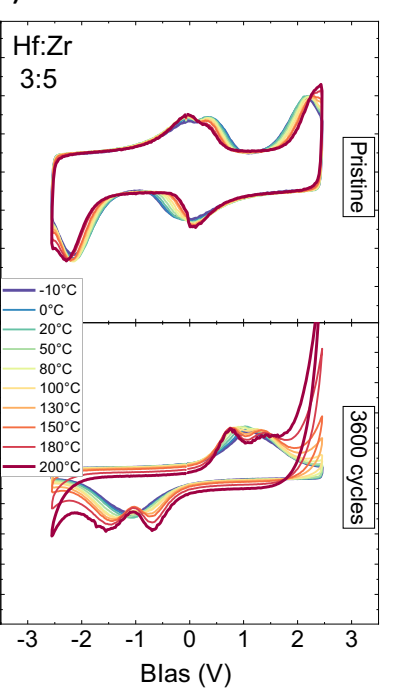

(b)

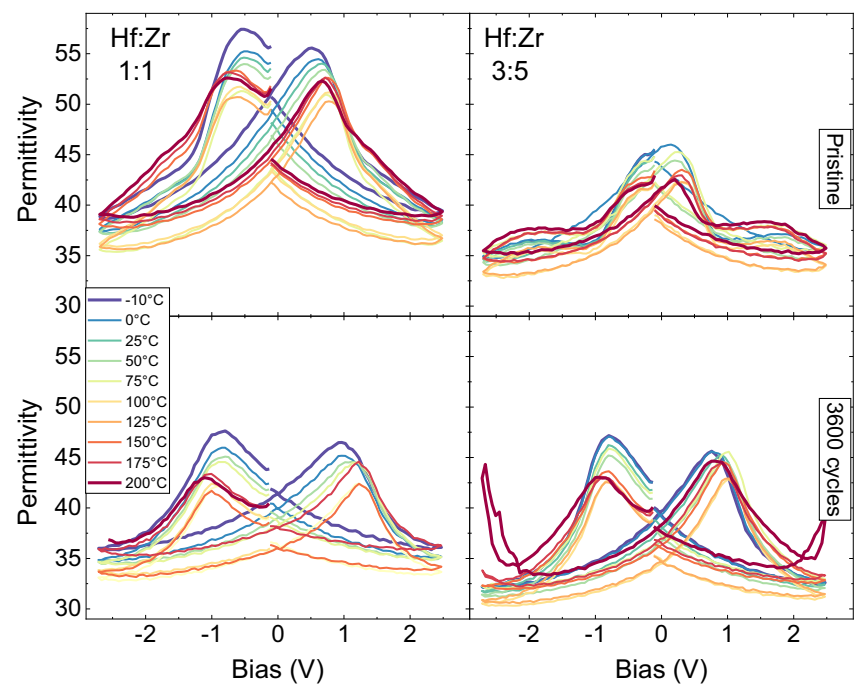

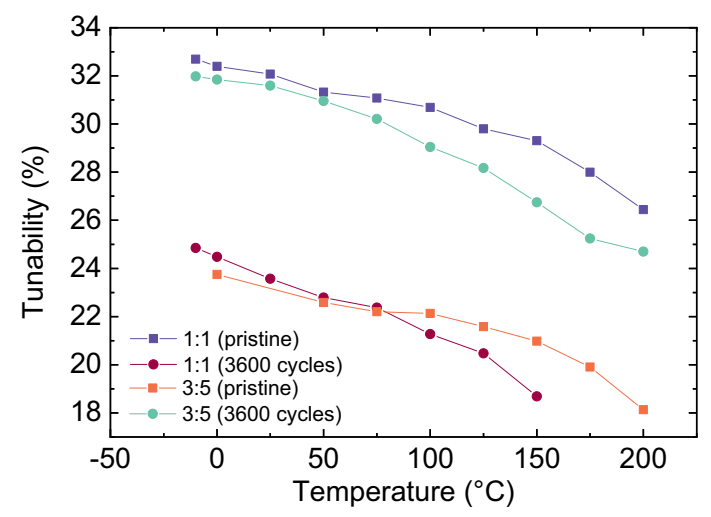

(c)

Fig. 4 I-V (a), C-V (b) characteristics, and tunability (c) of HZO with 1:1 and 3:5 Hf:Zr doping ratios at different temperatures, before and after the cycling

In case of HZO film with a doping ratio of 3:5, the $V_{c}$ peak is located close to zero in the pristine state. This effect can be useful for varactor applications, since a smaller voltage range is needed for capacitance tuning (Fig. 3c), which results in a faster tuning. Furthermore, in Fig. 3b, it can be seen that upon cycling, the tunability of $1: 1$ doped HZO is deteriorating quickly, whereas in case of 3:5 doped HZO, it remains more stable. This also favors the HZO with 3:5 doping for varactor applications.

In Fig. 4a, a development of I-V characteristics upon elevated temperature before and after wake-up is presented. The temperature was hereby varied between -10 and 200 ${ }^{\circ} \mathrm{C}$. For pristine films of both 1:1 and 3:5 doped HZO, the effect of temperature manifests itself in a peak separation, which shows a reversal of the wake-up process. The same phenomena was already shown in $[10,11]$. In the case of $3: 5$ doping ratio, this effect can still be seen after cycling. The wake-up reversal comes into agreement with the ferroelastic switching, since with the increasing temperature, there is an increase in the in-plane stress, which makes the out-ofplane-oriented domains less energetically favorable.

In Fig. 4b, it can be seen that increasing temperature also leads to the peak separation on the $\mathrm{C}-\mathrm{V}$ curves. With an increase in temperature, the tuning ranges of both $1: 1$ and 3:5 doped capacitors decrease. In Fig. 4c, the tunability values are extracted from the $\mathrm{C}-\mathrm{V}$ curves and plotted with temperature. In all the cases, there is a similar rate of decrease of tunability.

\section{Conclusion}

In this work, $\mathrm{HZO}$ capacitors for varactor applications, with different Hf:Zr doping ratios were analyzed. It was shown that the HZO films with 3:5 Hf:Zr doping, having a predominant AFE behavior, is a better candidate for varactor 
applications, since it requires lower tuning voltages and has a more stable tunability with cycling. With an increase in temperature, the tunability of both 1:1 and 3:5 doped varactors decreases with a similar rate. It was also shown that with an increasing temperature, a wake-up reversal can be observed, which leads to a peak separation in the I-V characteristics. This is in agreement with the latest studies of the ferroelastic switching as a main trigger for the antiferroelectric-like behavior in ferroelectric hafnium oxide.

Funding Open Access funding enabled and organized by Projekt DEAL. The current work was financed by the German Bundesministerium für Wirtschaft (BMWi) and by the State of Saxony, in the frame of projects WIN FDSOI from Important Project of Common European Interest (IPCEI). This study was supported by Fraunhofer-Gesellschaft (Grant No. 335061).

Data availability Research data in this article are not shared.

\section{Declarations}

Conflict of interest The authors declare that they have no conflict of interest.

Open Access This article is licensed under a Creative Commons Attribution 4.0 International License, which permits use, sharing, adaptation, distribution and reproduction in any medium or format, as long as you give appropriate credit to the original author(s) and the source, provide a link to the Creative Commons licence, and indicate if changes were made. The images or other third party material in this article are included in the article's Creative Commons licence, unless indicated otherwise in a credit line to the material. If material is not included in the article's Creative Commons licence and your intended use is not permitted by statutory regulation or exceeds the permitted use, you will need to obtain permission directly from the copyright holder. To view a copy of this licence, visit http://creativecommons.org/licenses/by/4.0/.

\section{References}

1. N.K. Pervez, P.J. Hansen, R.A. York, High tunability barium strontium titanate thin films for rf circuit applications. Appl. Phys. Lett. 85(19), 4451 (2004). https://doi.org/10.1063/1.1818724

2. T.S. Böscke, P.Y. Hung, P.D. Kirsch, M.A. Quevedo-Lopez, R. Ramírez-Bon, Dipole model explaining high-k/metal gate field effect transistor threshold voltage tuning. Appl. Phys. Lett. 95(5), 052904 (2009)

3. D. Lehninger, R. Olivo, T. Ali, M. Lederer, T. Kämpfe, C. Mart, K. Biedermann, K. Kühnel, L. Roy, M. Kalkani, K. Seidel, Backend-of-line compatible low-temperature furnace anneal for ferroelectric hafnium zirconium oxide formation. physica status solidi (a) 217(8), 1900840 (2020). https://doi.org/10.1002/pssa.20190 0840

4. M. Dragoman, M. Aldrigo, M. Modreanu, D. Dragoman, Extraordinary tunability of high-frequency devices using $\mathrm{Hf0}$.3Zr0.7O2 ferroelectric at very low applied voltages. Appl. Phys. Lett. 110(10), 103104 (2017). https://doi.org/10.1063/1.4978032

5. S. Abdulazhanov, Q.H. Le, D.K. Huynh, D. Wang, G. Gerlach, T. Kämpfe, A mmwave phase shifter based on ferroelectric hafnium zirconium oxide varactors. IEEE MTT-S International Microwave Workshop Series on Advanced Materials and Processes for RF and THz Applications (IMWS-AMP) 2019 Jul 16 (pp. 175-177)

6. S. Abdulazhanov, Q.H. Le, D.K. Huynh, D. Wang, G. Gerlach, T. Kämpfe, A tunable mmwave band-pass filter based on ferroelectric hafnium zirconium oxide varactors. IEEE MTT-S International Microwave Workshop Series on Advanced Materials and Processes for RF and THz Applications (IMWS-AMP) $2019 \mathrm{Jul}$ 16 (pp. 46-48)

7. D. Zhou, J. Xu, Q. Li, Y. Guan, F. Cao, X. Dong, J. Müller, T. Schenk, U. Schröder, Wake-up effects in Si-doped hafnium oxide ferroelectric thin films. Appl. Phys. Lett. 103(19), 192904 (2013)

8. M. Lederer, T. Kämpfe, R. Olivo, D. Lehninger, C. Mart, S. Kirbach, T. Ali, P. Polakowski, L. Roy, K. Seidel, Local crystallographic phase detection and texture mapping in ferroelectric $\mathrm{Zr}$ doped $\mathrm{HfO}_{2}$ films by transmission-EBSD. Appl. Phys. Lett. 115(22), 222902 (2019). https://doi.org/10.1063/1.5129318

9. M. Lederer, T. Kämpfe, N. Vogel, D. Utess, B. Volkmann, T. Ali, R. Olivo, J. Müller, S. Beyer, M. Trentzsch, K. Seidel, Structural and electrical comparison of $\mathrm{Si}$ and $\mathrm{Zr}$ doped hafnium oxide thin films and integrated FeFETs utilizing transmission kikuchi diffraction. Nanomaterials. 10(2), 384 (2020)

10. M. Lederer, R. Olivo, D. Lehninger, S. Abdulazhanov, T. Kämpfe, S. Kirbach, C. Mart, K. Seidel, L.M. Eng, On the origin of wakeup and antiferroelectric-like behavior in ferroelectric hafnium oxide. physica status solidi (RRL)-Rapid Research Letters. 15(5):2100086 (2021)

11. C. Mart, S. Abdulazhanov, M. Czernohorsky, T. Kämpfe, D. Lehninger, K. Falidas, S. Eßlinger, K. Kühnel, S. Oehler, M. Rudolph, M. Wiatr, Energy harvesting in the back-end of line with CMOS compatible ferroelectric hafnium oxide. IEEE International Electron Devices Meeting (IEDM) 2020 Dec 12 (pp. 26-3) 